\title{
Revealing the system-bath coupling via Landau-Zener-Stückelberg interferometry in superconducting qubits
}

\author{
Ana Laura Gramajo, ${ }^{1}$ Daniel Domínguez, ${ }^{1}$ and María José Sánchez ${ }^{1,2}$ \\ ${ }^{1}$ Centro Atómico Bariloche and Instituto Balseiro, 8400 San Carlos de Bariloche, Río Negro, Argentina \\ ${ }^{2}$ Instituto de Nanociencia y Nanotecnología, CONICET-CNEA, 8400 San Carlos de Bariloche, Río Negro, Argentina
}

(Received 12 June 2019; published 5 August 2019)

\begin{abstract}
In this work we propose a way to unveil the type of environmental noise in strongly driven superconducting flux qubits through the analysis of the Landau-Zener-Stückelberg (LZS) interferometry. We study both the two-level and multilevel dynamics of the flux qubit driven by a $\mathrm{dc}+\mathrm{ac}$ magnetic field. We find that the LZS interference patterns exhibit well-defined multiphoton resonances whose shape strongly depends on the timescale and the type of coupling to a quantum bath. For the case of transverse system-bath coupling, the $n$-photon resonances are narrow and nearly symmetric with respect to the dc magnetic field for almost all timescales, while in the case of longitudinal coupling they exhibit a change from a wide symmetric to an antisymmetric shape for times of the order of the relaxation time. We find this dynamic behavior relevant for the interpretation of several LZS interferometry experiments in which the stationary regime is not completely reached.
\end{abstract}

DOI: 10.1103/PhysRevB.100.075410

\section{INTRODUCTION}

Superconducting circuits with Josephson junctions [1,2] behave as artificial atoms [3] and have been extensively proven as quantum bits [4]. When driven by a dc + ac magnetic flux, Landau-Zener-Stückelberg (LZS) interference patterns [5] combined with multiphoton resonances have been observed [6-10] and used to probe the energy level spectrum of the device for large driving amplitudes $[8,9]$. LZS patterns also emerge in charge qubits [11,12], Rydberg atoms [13], ultracold molecular gases [14], optical lattices [15], and single-electron spins systems [16]. In addition, LZS interferometry was recently proposed as a tool to determine relevant information related to the coupling of a qubit with a noisy environment, such as dissipation strength and dephasing time [17-19]. These studies have been performed for steady-state experiments, where full relaxation with the bath degrees of freedom is assumed.

In the present work we demonstrate that the finite-time LZS spectroscopy can unveil additional features linked to how relevant timescales affect the symmetry of the resonance patterns for different system-bath couplings. As a system of study we chose the superconducting flux qubit (FQ) originally introduced in Ref. [1] which, in recent years, due to the improvement in its design and fabrication techniques, has become one of the most tested devices for quantum information proposals [20]. Recent experiments on the FQ have implemented noise spectroscopy for different sources of noise (flux noise, charge noise, critical current noise) through dynamical decoupling [21] and driven evolution measurements [22,23].

Here to address the finite-time LZS interferometry we study the FQ coupled to a quantum bath under strong periodic driving, using the Floquet-Markov quantum master equations [24,25]. Our main finding is that a dynamic change in the symmetry of an $n$-photon resonance takes place for the case of longitudinal system-bath coupling, while the resonances remain almost undisturbed in time for transverse system-bath coupling.

Our analysis becomes particularly relevant to understand LZS interferometry experiments for FQs with large relaxation times $[6,9]$. Several well-established theoretical works have studied the steady state of periodically driven two-level systems [26-30]. However, the experimental results on LZS interferometry in the FQ do not agree with these previous theoretical results. The theory of [26-30] shows population inversion and antisymmetric resonance patterns as a function of the energy detuning, instead of the symmetric patterns observed in the FQ experiments [6,9]. A possible explanation was put forward in Refs. [31,32]: there is a dynamic transition from symmetric resonance patterns below the relaxation time $t_{r}$ to antisymmetric resonance patterns for timescales above $t_{r}$. Since the FQ experiments were performed at finite timescales below $t_{r}$, the steady-state patterns were not observed, according to this scenario. On the other hand, in Ref. [18] it was shown that transverse noise (previous works [26-32] considered longitudinal noise) can lead to steady-state symmetric resonances in LZS interferometry, which suggest a different possible explanation of the experimental results. The aim of this work is to assess which scenario is more adequate to explain the experiments of Refs. [6,9] by analyzing the time dependence of the LZS patterns for different system-bath couplings (transverse and longitudinal noise).

We start in Sec. I by writing the Hamiltonian of the FQ in the presence of different sources of quantum noise and describing the Floquet-Markov formulation for open quantum systems with a time-periodic drive. In Sec. II we show results for the time-dependent evolution of the driven FQ with different sources of noise, restricted to a two-level system (TLS) regime. In Sec. III we extend the analysis to the multilevel case, which is relevant for large driving amplitudes and to compare with LZS experiments. Conclusions are given in Sec. IV. 


\section{DYNAMICS OF THE FLUX QUBIT}

\section{A. The flux qubit and noise sources}

The FQ consists of a superconducting ring with three Josephson junctions [1] enclosing a magnetic flux $\Phi=f \Phi_{0}$ $\left(\Phi_{0}=h / 2 e\right)$ with phase differences $\varphi_{1}, \varphi_{2}$, and $\varphi_{3}=-\varphi_{1}+$ $\varphi_{2}-2 \pi f$. Two of the junctions have coupling energy $E_{J}$ and capacitance $C$, while the third has $E_{J, 3}=\alpha E_{J}$ and $C_{3}=\alpha C$. In the quantum regime, the FQ Hamiltonian reads [1]

$$
\mathcal{H}_{\mathrm{FQ}}=E_{p} n_{p}^{2}+E_{m} n_{m}^{2}+E_{J} V,
$$

with $\varphi_{p}=\frac{\varphi_{1}+\varphi_{2}}{2}$ and $\varphi_{m}=\frac{\varphi_{1}-\varphi_{2}}{2}$ being the phase operators, $n_{k}=-i \frac{\partial}{\partial \varphi_{k}}(k=p, m)$ being the charge number operators, $E_{p}=2 E_{C}, E_{m}=\frac{E_{p}}{1+2 \alpha}, E_{C}=e^{2} / 2 C$, and $V\left(\varphi_{p}, \varphi_{m} ; f\right)=2+$ $\alpha-2 \cos \varphi_{p} \cos \varphi_{m}-\alpha \cos \left(2 \pi f+2 \varphi_{m}\right)$. The FQ has several levels with eigenenergies $E_{i}$ and eigenstates $\left|\Psi_{i}\right\rangle$ which depend on $\alpha, \eta=\sqrt{8 E_{C} / E_{J}}$ and flux detuning $\tilde{f}=f-1 / 2$. Typical experiments have $\alpha \sim 0.6-0.9$ and $\eta \sim 0.1-0.6[2$, 6-9]. For $\alpha \geqslant 1 / 2$ and $|\tilde{f}| \ll 1$, the potential $V$ has the shape of a double well with two minima along the $\varphi_{l}$ direction. Each minimum corresponds to macroscopic persistent currents of opposite sign, and for $\tilde{f} \gtrsim 0(\tilde{f} \lesssim 0)$ a ground state with positive (negative) loop current is favored. In this regime the system can be operated as a quantum bit [1,2] and approximated by a TLS $[1,33]$.

The main sources of relaxation and decoherence in the FQ are flux noise $\delta f(t)$, charge noise $\delta N(t)$, and critical current noise $\delta I_{c}(t)[21-23,34,35]$. In the case of weak fluctuations, the different sources of noise can be incorporated in Eq. (1) by the replacements $f \rightarrow f+\delta f, n_{k} \rightarrow n_{k}-\delta N_{k}(k=p, m)$, and $E_{J} \rightarrow E_{J}\left(1+\delta I_{c} / I_{c}\right)$, respectively [21-23,34]. This leads to $\mathcal{H}_{\mathrm{FQ}} \rightarrow \mathcal{H}_{\mathrm{FQ}}^{\prime} \approx \mathcal{H}_{\mathrm{FQ}}+H_{\mathrm{int}}$, where

$$
H_{\mathrm{int}}=H_{\mathrm{int}}^{c h}+H_{\mathrm{int}}^{f}+H_{\mathrm{int}}^{c c}
$$

and

$$
\begin{aligned}
H_{\mathrm{int}}^{c h} & =-2 E_{p} n_{p} \delta N_{p}-2 E_{m} n_{m} \delta N_{m}, \\
H_{\mathrm{int}}^{f} & =-2 \pi E_{J} I \delta f, \\
H_{\mathrm{int}}^{c c} & =\frac{E_{J}}{I_{c}} V \delta I_{c},
\end{aligned}
$$

with $I=\alpha \sin \left(2 \pi \tilde{f}+2 \varphi_{m}\right)$, the loop current operator normalized by $I_{c}=\frac{2 \pi E_{J}}{\Phi_{0}}$. Notice that Eq. (2) results from neglecting quadratic terms in $\left(n_{p}-N_{p}\right)^{2}$ and $\left(n_{m}-N_{m}\right)^{2}$ since we are assuming the weak-fluctuation regime.

If we consider the lowest eigenstates, the term with $n_{p}$ can be neglected [36], and we can redefine the system-bath interaction Hamiltonian as

$$
H_{\mathrm{int}}=\mathcal{A}^{c h} \otimes \mathcal{B}^{c h}+\mathcal{A}^{f} \otimes \mathcal{B}^{f}+\mathcal{A}^{c c} \otimes \mathcal{B}^{c c},
$$

where the system operators are $\mathcal{A}^{\text {ch }}=-2 E_{m} n_{m}, \mathcal{A}^{f}=$ $-2 \pi E_{J} I, \mathcal{A}^{c c}=E_{J} V$ and the normalized bath (noise) operators are $\mathcal{B}^{c h}=\delta N_{p}, \mathcal{B}^{f}=\delta f$, and $\mathcal{B}^{c c}=\delta I_{c} / I_{c}$.

As a first approach we will consider in Sec. II the FQ restricted to the two lowest computational levels [1,33],

$$
\mathcal{H}_{\mathrm{TLS}}=-\frac{\epsilon}{2} \sigma_{z}-\frac{\Delta}{2} \sigma_{x},
$$

where the Hamiltonian is written in the basis defined by the persistent current states $|+\rangle=(|0\rangle+|1\rangle) / \sqrt{2}$ and $|-\rangle=$ $(|0\rangle-|1\rangle) / \sqrt{2}$, where $|0\rangle$ and $|1\rangle$ are the ground and excited FQ states at $\delta f=0$. The parameters of $\mathcal{H}_{\mathrm{TLS}}$ are the detuning $\epsilon=4 \pi I_{p} \tilde{f}$ and the energy gap $\Delta=E_{1}-E_{0}$ at $\tilde{f}=0$. Here $I_{p}=|\langle+|I|+\rangle|=|\langle-|I|-\rangle|$ is the magnitude of the loop current. Within this approximation, the noise coupling operators become

$$
\mathcal{A}^{f}=-\lambda_{f} \sigma_{z}, \quad \mathcal{A}^{c h}=-\lambda_{c h} \sigma_{y}, \quad \mathcal{A}^{c c}=-\lambda_{c c} \sigma_{x},
$$

with $\lambda_{f}=2 \pi|\langle+|I|+\rangle|, \lambda_{c h}=\frac{\eta^{2}}{4+8 \alpha}\left|\left\langle-\left|n_{m}\right|+\right\rangle\right|$, and $\lambda_{c c}=$ $-|\langle-|V|+\rangle|$.

For the parameter values $\alpha=0.8$ and $\eta=0.25$ and after diagonalization of $\mathcal{H}_{\mathrm{TLS}}$, we obtain $I_{p}=0.721$ (in units of $E_{J} / \Phi_{0}$ ) and $\Delta=3.33 \times 10^{-4}$ (in units of $E_{J}$ ). Thus, the noise coupling parameters result in $\lambda_{f} \approx 4.5, \lambda_{c h} \approx 3 \times 10^{-4}$, and $\lambda_{c c} \approx 4 \times 10^{-3}$ (the neglected term corresponding to $n_{p}$ has the coupling parameter $\lambda_{c h}^{p}=\frac{\eta^{2}}{4}\left|\left\langle-\left|n_{p}\right|+\right\rangle\right| \approx 10^{-13}$ ).

\section{B. LZS interferometry in the presence of quantum noise: The Floquet-Markov approach}

In experiments with flux qubits, LZS interferometry $[6-9,37]$ is performed by applying a harmonic (ac) field of frequency $\omega_{0}$ on top of the static flux, i.e.,

$$
\tilde{f} \rightarrow \tilde{f}(t)=\tilde{f}_{\mathrm{dc}}+f_{\mathrm{ac}} \cos \left(\omega_{0} t\right) .
$$

In this work, following Refs. [24,30-32,38-41], we analyze the LZS interferometry employing the Floquet formalism, which allows for an exact treatment of harmonic drivings of arbitrary strength and frequency. Alternative approaches to the description of the LZS interference patterns rely on approximations valid either for large driving frequencies or low driving amplitudes [5,7,42].

For the harmonic driving, the Hamiltonian of the FQ results in time-periodic $\mathcal{H}_{\mathrm{FQ}}(t)=\mathcal{H}_{\mathrm{FQ}}(t+\tau)$, with $\tau=2 \pi / \omega_{0}$. In the Floquet formalism, the solutions of the Schrödinger equation are of the form $\left|\Psi_{\alpha}(t)\right\rangle=e^{i \varepsilon_{\alpha} t / \hbar}|\alpha(t)\rangle$, where the Floquet states $|\alpha(t)\rangle$ satisfy $|\alpha(t)\rangle=|\alpha(t+\tau)\rangle=\sum_{k}\left|\alpha_{k}\right\rangle e^{-i k \omega t}$, and are eigenstates of the equation $[\mathcal{H}(t)-i \hbar \partial / \partial t]|\alpha(t)\rangle=$ $\varepsilon_{\alpha}|\alpha(t)\rangle$, with $\varepsilon_{\alpha}$ being the associated quasienergy.

Since the FQ is in contact with the environment, the total Hamiltonian of the open system is

$$
H=H_{s}(t)+H_{B}+H_{\text {int }} .
$$

Here $H_{S}$ is the system Hamiltonian (in our case $H_{s}=$ $\left.\mathcal{H}_{\mathrm{FQ}}\right), H_{B}$ is the Hamiltonian of the environment, which is usually modeled as a bath of harmonic oscillators [25,29,30,39,40,43,44], and $H_{\text {int }}$ is the system-bath interaction Hamiltonian. For weak coupling (Born approximation) and fast bath relaxation (Markov approximation), a FloquetBorn-Markov master equation for the system reduced density matrix $\rho$ in the Floquet basis, $\rho_{\alpha \beta}(t)=\langle\alpha(t)|\rho(t)| \beta(t)\rangle$, can be obtained $[25,39,40]$ :

$$
\begin{aligned}
\frac{d \rho_{\alpha \beta}(t)}{d t} & =\sum_{\alpha^{\prime} \beta^{\prime}} \Lambda_{\alpha \beta \alpha^{\prime} \beta^{\prime}} \rho_{\alpha^{\prime} \beta^{\prime}}, \\
\Lambda_{\alpha \beta \alpha^{\prime} \beta^{\prime}} & =-\frac{i}{\hbar}\left(\varepsilon_{\alpha}-\varepsilon_{\beta}\right) \delta_{\alpha \alpha^{\prime}} \delta_{\beta \beta^{\prime}}+L_{\alpha \beta \alpha^{\prime} \beta^{\prime}} .
\end{aligned}
$$


The coefficients $L_{\alpha \beta \alpha^{\prime} \beta^{\prime}}$ are usually rewritten in terms of transition rates $R_{\alpha \beta \alpha^{\prime} \beta^{\prime}}$ as

$$
\begin{aligned}
L_{\alpha \beta \alpha^{\prime} \beta^{\prime}}= & R_{\alpha \beta \alpha^{\prime} \beta^{\prime}}+R_{\beta \alpha \beta^{\prime} \alpha^{\prime}}^{*} \\
& -\sum_{\eta}\left(\delta_{\beta \beta^{\prime}} R_{\eta \eta \alpha^{\prime} \alpha}+\delta_{\alpha \alpha^{\prime}} R_{\eta \eta \beta^{\prime} \beta}^{*}\right) .
\end{aligned}
$$

As we already showed in Eq. (4), the interaction Hamiltonian can be written as

$$
H_{\text {int }}=\sum_{v} \mathcal{A}^{v} \otimes \mathcal{B}^{v},
$$

where $\mathcal{A}^{v}$ are system operators and $\mathcal{B}^{v}$ are bath operators associated with different noise sources. In the case of independent noise sources, the corresponding bath operators are uncorrelated, such that $\left\langle\mathcal{B}^{v}(t) \mathcal{B}^{v^{\prime}}\left(t^{\prime}\right)\right\rangle=0$ for $v \neq v^{\prime}$, and the transition rates $R_{\alpha \beta \alpha^{\prime} \beta^{\prime}}$ are given as

$$
R_{\alpha \beta \alpha^{\prime} \beta^{\prime}}=\sum_{\nu} R_{\alpha \beta \alpha^{\prime} \beta^{\prime}}^{v},
$$

with

$$
R_{\alpha \beta \alpha^{\prime} \beta^{\prime}}^{v}=\sum_{q} g^{\nu}\left(\omega_{\alpha \alpha^{\prime}, q}\right) A_{\alpha \alpha^{\prime}, q}^{v} A_{\beta^{\prime} \beta,-q}^{v},
$$

where $\omega_{\alpha \alpha^{\prime}, q}=\left(\varepsilon_{\alpha}-\varepsilon_{\alpha}^{\prime}\right) / \hbar+q \omega_{0}$. In this way, the systembath interaction is encoded in the transition matrix elements

$$
A_{\alpha \beta, q}^{v}=\sum_{k}\left\langle\alpha_{k}\left|\mathcal{A}^{v}\right| \beta_{k+q}\right\rangle .
$$

Assuming that each bath is in equilibrium at temperature $T^{v}$, it is customary to define

$$
g^{\nu}(\omega)=\int_{-\infty}^{\infty} d t\left\langle\mathcal{B}^{\nu}(t) \mathcal{B}^{\nu}(0)\right\rangle e^{-i \omega t}=J^{v}(\omega) n_{\mathrm{th}}^{v}(\hbar \omega),
$$

where $J^{\nu}(\omega)$ [defining $J^{\nu}(-\omega)=-J^{\nu}(\omega)$ ] is the bath spectral density and $n_{\mathrm{th}}^{\nu}(\varepsilon)=1 /\left[\exp \left(\varepsilon / k_{B} T^{\nu}\right)-1\right]$ [45].

In this work, we numerically compute the Floquet states $|\alpha(t)\rangle$ and calculate the coefficients $L_{\alpha \beta \alpha^{\prime} \beta^{\prime}}$, from which the solution of $\rho_{\alpha \beta}(t)$ can be obtained $[32,41]$.

\section{TWO-LEVEL REGIME}

\section{A. Unitary evolution and LZS interferometry}

We start by reviewing the LZS interferometry for the TLS. In order to obtain the driven Hamiltonian, we replace in $\mathcal{H}_{\mathrm{TLS}}$

$$
\epsilon \rightarrow \epsilon(t)=\epsilon_{0}+A \cos \left(\omega_{0} t\right),
$$

with $\epsilon_{0}=4 \pi I_{p} \tilde{f}_{\mathrm{dc}}$ and $A=4 \pi I_{p} f_{\mathrm{ac}}$. The frequency $\omega_{0}$ is written in units of $E_{J} / \hbar$, and the qubit eigenenergies are written in units of $E_{J}$. When $f_{\mathrm{ac}}>\left|\tilde{f}_{\mathrm{dc}}\right|$, the central avoided crossing at $\tilde{f}=0$ is reached for driving amplitudes $\tilde{f}_{\mathrm{dc}} \pm f_{\mathrm{ac}}$. In this case the periodically repeated transitions at $\tilde{f}=0$ give rise to the LZS interference patterns as a function of $\tilde{f}_{\mathrm{dc}}$ and $f_{\mathrm{ac}}$, characterized by multiphoton resonances at $E_{1}(\tilde{f})-E_{0}(\tilde{f})=$ $n \omega_{0}[5,24,30,39,40,42]$.

For $\epsilon_{0} \gg \Delta$ the resonances take place at $\epsilon_{0} \simeq n \omega_{0}$, and denoting $f_{\omega}=\omega_{0} / 4 \pi I_{p}$, the $n$-resonance condition can be written as $\epsilon_{0} / \omega_{0}=\tilde{f}_{\mathrm{dc}} / f_{\omega}=n$.

In the regime $A \omega_{0} \gg \Delta^{2}$ and in the rotating wave approximation (RWA) [5,7,42], the time-averaged probability of measuring a positive loop current $\overline{P_{+}}=\overline{|\langle\Psi(t) \mid+\rangle|^{2}}$ near an $n$-photon resonance is

$$
\overline{P_{+}}=1-\frac{1}{2} \frac{\Delta_{n}^{2}}{\left(n \omega_{0}-\epsilon_{0}\right)^{2}+\Delta_{n}^{2}} .
$$

When the resonance condition $\epsilon_{0}=n \omega_{0}$ is satisfied, Eq. (12) gives $\overline{P_{+}}=1 / 2$; otherwise, it gives $\overline{P_{+}} \lesssim 1$. Furthermore, the width of the resonance is $\delta \epsilon=\left|\Delta_{n}\right|=\Delta\left|J_{n}\left(A / \omega_{0}\right)\right|=$ $\Delta\left|J_{n}\left(f_{\text {ac }} / f_{\omega}\right)\right|$, with $J_{n}(x)$ being the Bessel function of the first kind. This gives a quasiperiodic dependence as a function of $f_{\text {ac }}$ for $\tilde{f}_{\text {dc }}$ fixed near the resonance. In particular, at the zeros of $J_{n}(x)$ the resonance is destroyed, with $\overline{P_{+}} \sim 1$ instead of $\overline{P_{+}}=1 / 2$, a phenomenon known as coherent destruction of tunneling $[46,47]$. Plots of $\overline{P_{+}}$as a function of flux detuning $\tilde{f}_{\mathrm{dc}}$ and ac amplitude $f_{\text {ac }}$ give the typical LZS interference patterns, which have been measured experimentally in flux qubits [6-9,37] and have also been observed in other driven systems [10-12,14-17,48-62].

Several phenomenological approaches $[3,5,8,63]$ have taken into account relaxation and decoherence effects in LZS interferometry, obtaining a broadening of the Lorentzianshape $n$-photon resonances of Eq. (12).

\section{B. Longitudinal vs transverse noise}

In this section we analyze the environmental noise employing the Floquet Markov master equation, described in Sec. II B. We first consider the two extreme cases: either pure flux, or "longitudinal" noise (which commutes with the driving), or pure charge noise, which we call "transverse" noise. For simplicity, we consider in both cases that the baths are equilibrated at the same temperature $T^{v}=T$ with an Ohmic spectral density $J^{\nu}(\omega)=\gamma \omega e^{-\omega / \omega_{c}}$. In the case of pure longitudinal noise we consider $\mathcal{A}^{f} \rightarrow \mathcal{A}=-\lambda_{f} \sigma_{z}$, while in the case of pure transverse noise we take $\mathcal{A}^{c h} \rightarrow \mathcal{A}=-\lambda_{c h} \sigma_{y}$. In order to establish a quantitative comparison among the two types of noise we first analyze the results for equal coupling strengths $\lambda^{(f)}=\lambda^{(c h)}=1$.

We use typical reported experimental values for FQs [6], $E_{J} / h \sim 300 \mathrm{GHz}$, driving microwave frequency $\omega_{0} / 2 \pi=0.003 E_{J} / \hbar \sim 900 \mathrm{MHz}$, and bath temperature $T=0.0014 E_{J} / k_{B}(\sim 20 \mathrm{mK})$, and we consider $\gamma=0.001$. Furthermore, in all the cases we are assuming that the FQ is initially prepared in its ground state $\left|\Psi_{0}\right\rangle$ of the static Hamiltonian $H_{0} \equiv \mathcal{H}_{\mathrm{FQ}}\left(\tilde{f}=\tilde{f}_{\mathrm{dc}}\right)$. Experimentally, the probability of having a state of positive or negative persistent current in the FQ is measured [2,6]. The probability of a positive current measurement can be calculated as $P_{+}(t)=\operatorname{Tr}\left[\Pi_{+} \rho(t)\right]$, with $\Pi_{+}=|+\rangle\langle+|$. For a static detuning $\tilde{f}_{\mathrm{dc}} \gtrsim 0$, the ground state has $P_{+}(0) \approx 1$.

In Figs. 1(a) and 1(b) and 1(c) and 1(d) we plot $P_{+}$for longitudinal and transverse couplings, respectively, as a function of the flux detuning $\tilde{f}_{\mathrm{dc}}$ for a fixed value of $f_{\mathrm{ac}}=0.003$. As a comparison, for both couplings we plot $\bar{P}_{+}$for the isolated case (without dissipation), where the $n$-photon resonances are clearly displayed as minima at $\epsilon_{0} / \omega_{0}=\tilde{f}_{\mathrm{dc}} / f_{\omega}=n$. For longitudinal coupling, Fig. 1(a) shows that for the timescales of FQ experiments [6] (we take here as a typical value $t_{\exp }=$ $1000 \tau)$, the behavior of $P_{+}$is similar to the isolated case, with 

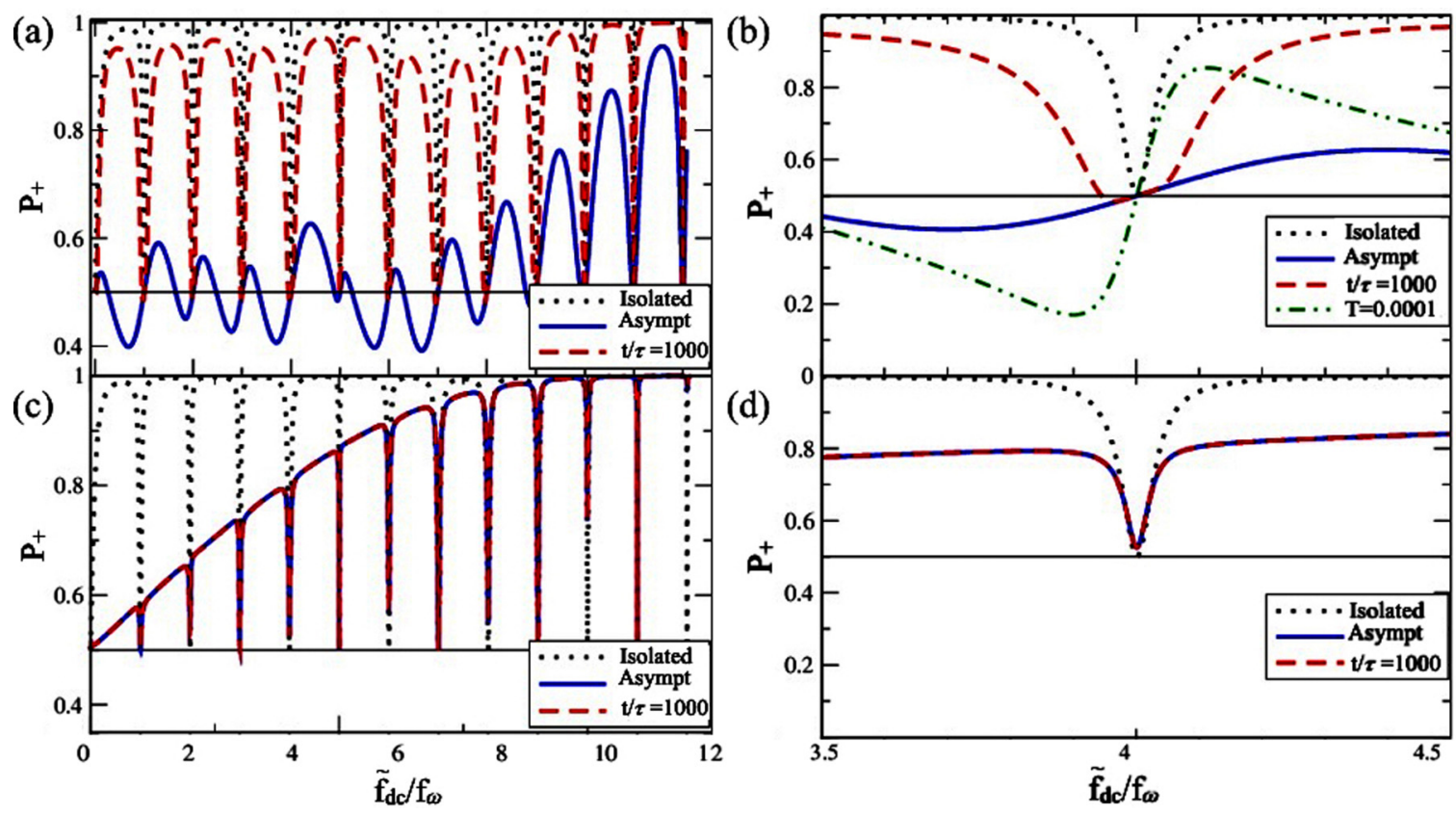

FIG. 1. Population $P_{+}$as a function of the dc flux detuning $\tilde{f}_{\mathrm{dc}}$ (normalized by $f_{\omega}=\omega_{0} / 4 \pi I_{p}$ ) for the FQ restricted to TLS, driven with amplitude $f_{\text {ac }}=0.003$ and $\omega_{0}=2 \pi / \tau=0.003 E_{J} / \hbar$. The Ohmic bath is at $T=0.0014$ (20 mK for $\left.E_{J} / h \approx 300 \mathrm{GHz}\right)$. Dotted line: $\bar{P}_{+}$for the isolated system; dashed line: $P_{+}(t=1000 \tau)$; solid line: asymptotic $(t \rightarrow \infty) \bar{P}_{+}$. The horizontal solid line indicates the $P_{+}=0.5$ value. (a) Longitudinal coupling for an Ohmic bath with $\gamma^{(f)}=0.001$ and (b) enlarged view of (a) around $n=4$ photon resonance. (c) Transverse coupling for an Ohmic bath with $\gamma^{(c h)}=0.001$ and (d) enlarged view of (c) around $n=4$ photon resonance.

a broadening of the minima at the multiphoton resonances due to decoherence. On the other hand, in the asymptotic $t \rightarrow \infty$ steady state, $P_{+}$exhibits antisymmetric multiphoton resonances [18,32], clearly displayed in Fig. 1(b), where an enlarged view around the $n=4$ resonance is shown. Moreover, as the temperature is lowered, the antisymmetry around the resonance condition is more evident, as shown for $T=$ $0.0001 E_{J} / k_{B}$.

For transverse coupling [see Figs. 1(c) and 1(d)], the behavior of $P_{+}$vs $\tilde{f}_{\mathrm{dc}}$ is remarkably different from the previous case: (i) there are no noticeable differences between the finite time and the steady sate $P_{+}$, (ii) the multiphoton resonances are symmetric in the steady state, (iii) there is no broadening of the resonances compared to the isolated case, and (iv) there is a linear background in $P_{+}$as a function of $\tilde{f}_{\mathrm{dc}}$ for the offresonant situations. Hallmarks (ii) and (iv) were also found in Ref. [18]. The linear background in $P_{+}$can be understood by a simple argument. The transverse coupling through $\sigma_{y}$ provides a direct relaxation mechanism to the ground state (the same holds for $\sigma_{x}$ coupling). Assuming that for the off-resonant situations in the steady state the qubit is fully relaxed in the ground state, we can estimate that on average $P_{+} \sim t^{\prime} / \tau$, with $t^{\prime}$ being the timescale within one period $\tau$ in which the ground state has a nearly complete overlap with the $|+\rangle$ state (when $\tilde{f}(t)>0$ ). For small $\tilde{f}_{\mathrm{dc}} / f_{\mathrm{ac}}$ this gives $P_{+} \sim \frac{1}{2}+$ $\frac{1}{\pi} \frac{\tilde{f}_{\mathrm{dc}}}{f_{\mathrm{ac}}}$. This straightforward calculation illustrates the linear background in the dependence of $P_{+}$with $f_{\mathrm{dc}}$ observed in Fig. 1(c).

The above-described features have a correlation in the behavior of the relaxation $\left(t_{r}\right)$ and the decoherence $\left(t_{d}\right)$ times, which are shown in Fig. 2 for both types of couplings. They are calculated numerically from the eigenvalues of $\Lambda$ defined in Eq. (8); the maximum nonzero real eigenvalue of $\Lambda$ gives $-t_{r}^{-1}$, and the real part of the complex-conjugate eigenvalues of $\Lambda$ gives $-t_{d}^{-1}[32,41]$. In general $\frac{1}{t_{d}}=\frac{1}{2 t_{r}}+\frac{1}{t_{\phi}}$, with $t_{\phi}$ being the dephasing time, and thus, the decoherence time satisfies $t_{d} \leqslant 2 t_{r}$ [24].

For the longitudinal coupling case we find in Fig. 2 that the equality $t_{d}=2 t_{r}$ is satisfied at the multiphoton resonances. Thus, at the resonances the dephasing mechanism vanishes, similar to what is usually found for the static case at the "sweet spot" $\tilde{f}=0[20,21]$. Away from resonances $t_{d} \ll t_{r}$, showing a large timescale separation between decoherence and relaxation due to strong dephasing. We have also obtained an analytic expression for the rates $\Gamma_{r}=1 / t_{r}$ and the decoherence rate $\Gamma_{d}=1 / t_{d}$ employing a RWA for detunings near the $n$-photon resonance, $\tilde{f} \sim n f_{\omega}$, which are in good agreement with these numerical results (see the Appendix B for a detailed calculation). In the case of longitudinal noise, the relaxation rates can be estimated as

$$
\begin{aligned}
& \Gamma_{r}=\left|\lambda_{f} \sin (2 \varphi)\right|^{2}\left[g\left(-\Omega_{n}\right)+g\left(\Omega_{n}\right)\right], \\
& \Gamma_{d}=\frac{\Gamma_{r}}{2}+\left|\lambda_{f} \cos (2 \varphi)\right|^{2} g(0),
\end{aligned}
$$

with $\cos (2 \varphi)=\epsilon_{n} / \Omega_{n}, \sin (2 \varphi)=\Delta_{-n} / \Omega_{n}, \epsilon_{n}=4 \pi I_{p}(\tilde{f}-$ $\left.n f_{\omega}\right)$. The generalized Rabi frequency is $\Omega_{n}=\sqrt{\epsilon_{n}^{2}+\Delta_{-n}^{2}}$, and $\Delta_{-n}=\Delta J_{-n}(x)$, with $x \equiv f_{\text {ac }} / f_{\omega}$. At the resonance $\cos (2 \varphi)=0$, and $\sin (2 \varphi)=1$; thus, $\Gamma_{d}=\Gamma_{r} / 2$, and $\Gamma_{r}$ is maximum. Away from resonance the dephasing rate is maximum, and $\Gamma_{\phi}=\Gamma_{d}-\Gamma_{r} / 2 \sim \lambda_{f}^{2} g(0) \approx \lambda_{f}^{2} \gamma k T$ [assuming $\cos (2 \varphi) \sim 1]$. This in agreement with the exact numerical 

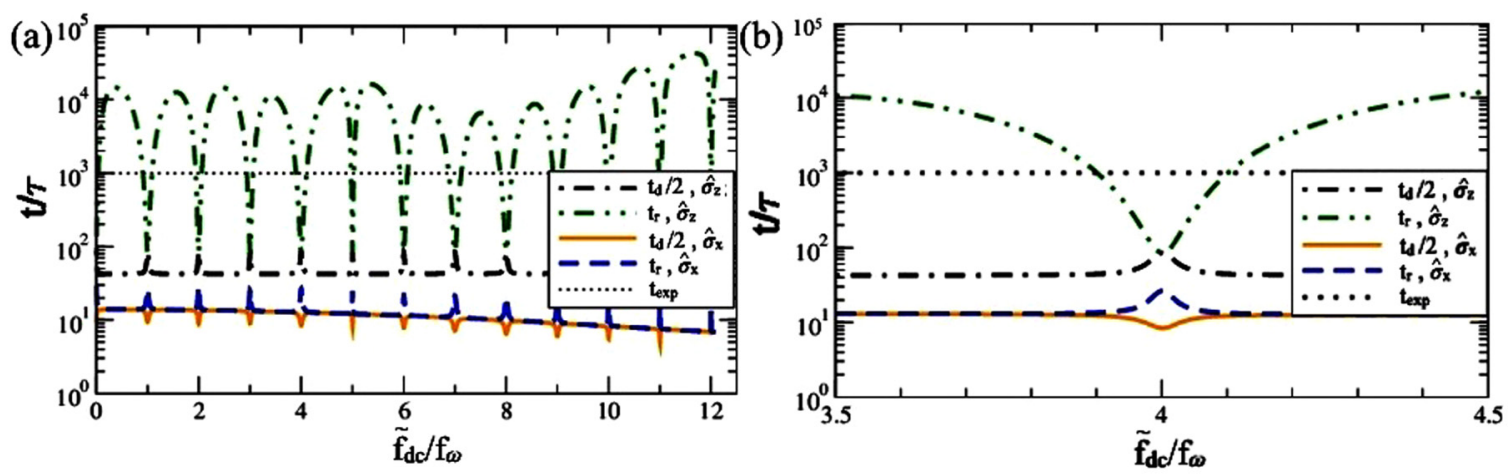

FIG. 2. (a) Relaxation time $t_{r}$ for the longitudinal (dash-double-dotted line) and transverse (dashed line) couplings. (Half) decoherence time $t_{d} / 2$ for the longitudinal (dash-dotted line) and transverse (solid line) couplings. The flux detuning is normalized by $f_{\omega}=\omega_{0} / 4 \pi I_{p}$, such that the $n$-photon resonances are at $\tilde{f}=n f_{\omega}$. (b) Enlarged view of (a) around the $n=4$ resonance. The experimental time $t_{\exp } / \tau=1000$ is plotted by the dotted line.

results of Figs. 2(a) and 2(b), where $t_{d} \ll t_{\exp } \ll t_{r}$ away from resonance for the longitudinal case. This timescale separation allows the dynamic transition described in Ref. [32] and is also shown in Fig. 3(a). We see that while $P_{+}$remains symmetric around a resonance for $t_{d}<t<t_{r}$, there is a dynamic transition to the antisymmetric behavior for $t>t_{r}$.

On the other hand, for the transverse coupling case we find in Figs. 2(a) and 2(b) that the equality $t_{d}=2 t_{r}$ is reached out of resonance, i.e., the opposite of the longitudinal case, while near the resonances the (small) dephasing gives $t_{d} \lesssim 2 t_{r}$. The RWA calculation detailed in the Appendix A is also consistent

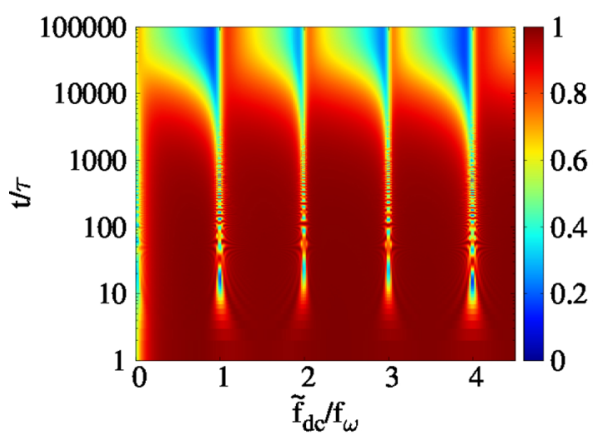

(a)

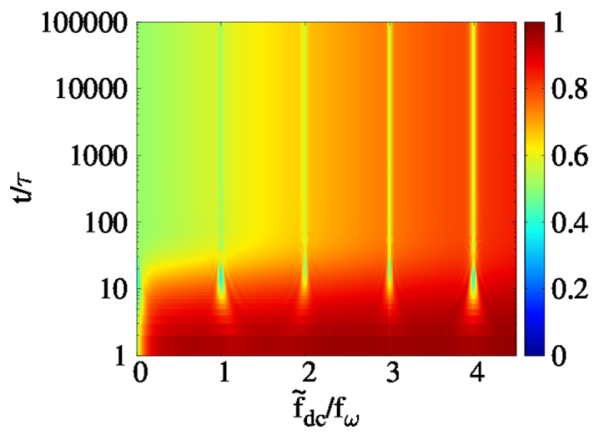

(b)

FIG. 3. Intensity plots of the population $P_{+}$as a function of $\tilde{f}_{\mathrm{dc}}$ and driving time $t$. (a) Longitudinal coupling for $\gamma^{(f)}=0.001$. (b) Transverse coupling for $\gamma^{(c h)}=0.001$. See text for details. with this numerical result. For the transverse coupling we got

$$
\begin{aligned}
& \Gamma_{r}=\left|\lambda_{c h} J_{-n}(x) \cos (2 \varphi)\right|^{2}\left[g\left(-\Omega_{n}\right)+g\left(\Omega_{n}\right)\right], \\
& \Gamma_{d}=\frac{\Gamma_{r}}{2}+\left|\lambda_{c h} J_{-n}(x) \sin (2 \varphi)\right|^{2} g(0) .
\end{aligned}
$$

In this case, away from resonance $\left|\epsilon_{n}\right| \gg \Delta_{-n}$, implying $\sin (2 \varphi) \sim 0, \cos (2 \varphi) \sim 1$, and thus $\Gamma_{d} \approx \Gamma_{r} / 2$. At resonance the opposite condition is satisfied: the dephasing rate is maximum, and thus, $\Gamma_{\phi} \propto\left|\lambda_{c h}\right|^{2} g(0) \approx\left|\lambda_{c h}\right|^{2} \gamma k T$.

In addition, for transverse coupling the system tends to relax fast to the steady state in comparison to the longitudinal coupling case (assuming the same coupling strengths $\lambda$ ). Note that, in the RWA calculation, out of resonance $\cos 2 \varphi^{2} \gg$ $\sin 2 \varphi^{2}$ and then $\Gamma_{r}^{\text {transverse }} \gg \Gamma_{r}^{\text {longitudinal }}$ for the same $\lambda$. This relatively fast relaxation is evident in Fig. 3(b), where the steady state is quickly reached and no symmetry change around the resonance is observed.

\section{Mixed noise}

We deal now with the more general case when two sources of independent noise are taken into account, as formulated in Sec. II, and we consider the two system-bath couplings with $\mathcal{A}^{f}=-\lambda_{f} \sigma_{z}$ and $\mathcal{A}^{c h}=-\lambda_{c h} \sigma_{y}$.

For simplicity we consider as before $J^{(f)}(\omega)=J^{(c h)}(\omega)=$ $\gamma \omega e^{-\omega / \omega_{c}}$.

In order to compare the relative coupling strengths we define $\lambda_{f}=\cos \theta$ and $\lambda_{c h}=\sin \theta$. We plot in Fig. $4 P_{+}$for $f_{\text {ac }}=0.003$ as a function of the mixing parameter $\cos ^{2} \theta$ and $\tilde{f}_{\mathrm{dc}}$ for the stationary case [Fig. 4(a)] and for finite time $t=1000 \tau$ [Fig. 4(b)]. In both cases the plots exhibit a behavior similar to the one obtained for the transverse coupling [see Fig. 3(b)] for almost the whole range of $\cos ^{2} \theta$. Only when $\left(\lambda^{(c h)} / \lambda^{(f)}\right)^{2}<0.005$ are the typical features of the pure longitudinal case (already described) observed.

In agreement with the observed response in $P_{+}$, Fig. 4(c) shows that $2 t_{r} / t_{d} \sim 1$ for almost the whole range of the mixing parameter, and only when approaching the longitudinal case, $\left(\lambda^{(c h)} / \lambda^{(f)}\right)^{2}<0.005$, is the timescale separation $2 t_{r} / t_{d} \gg 1$ observed in the off-resonant regions. 


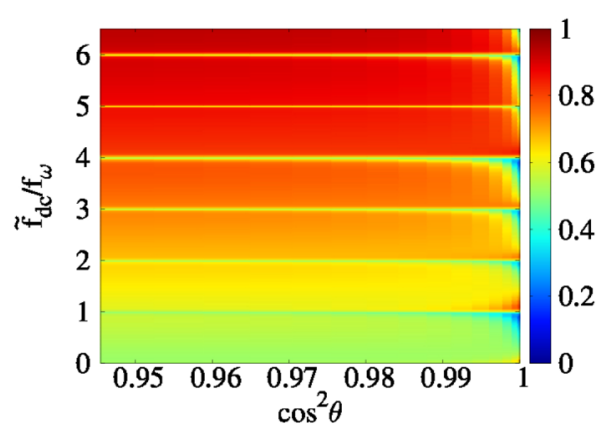

(a)

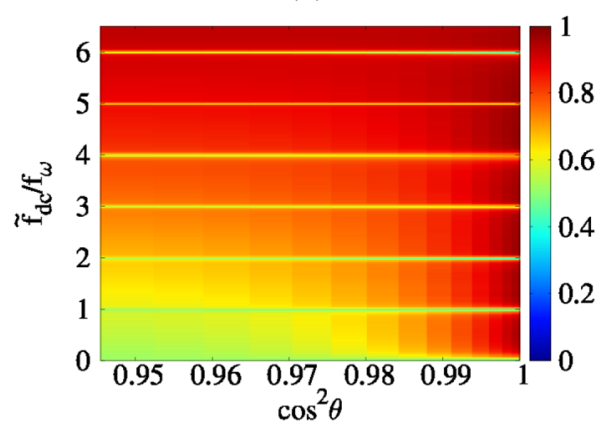

(b)

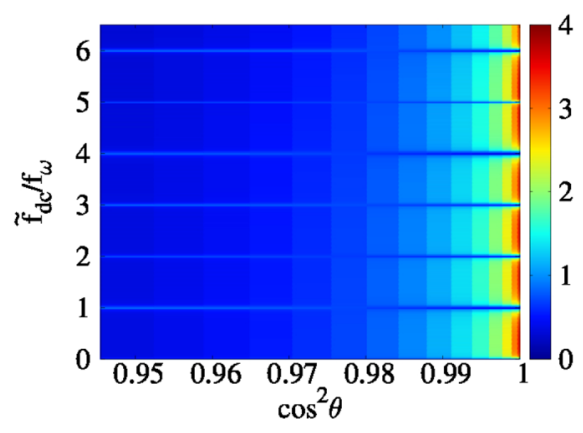

(c)

FIG. 4. Intensity plots of the population $P_{+}$as a function of $\tilde{f}_{\mathrm{dc}}$ and the mixing parameter $\cos ^{2} \theta \cdot \gamma^{(f)}=\gamma \cos ^{2} \theta$ and $\gamma^{(c h)}=$ $\gamma \sin ^{2} \theta$, with $\gamma=0.001$. (a) $t=\infty$, (b) $t=1000 \tau$. (c) Intensity plot of the ratio of $\log _{10}\left(2 t_{r} / t_{d}\right)$.

\section{MULTILEVEL REGIME: LZS DIAMONDS}

The previous analysis can be extended to the multilevel regime which corresponds to realistic parameters of the FQ. We focus on the dynamics of the four lowest energy levels of the device, where the spectrum shows a rich structure of avoided crossings as a function of the dc detuning [6,41]. We solve the Floquet-Markov equations for the Hamiltonian of Eq. (1), restricted to the subspace spanned by the four lowest levels. Here we will compare the LZS patterns for pure flux noise and pure charge noise. In both cases we consider an Ohmic bath with spectral density $J(\omega)=\gamma \omega e^{-\omega / \omega_{c}}$ at temperature $T$ but different coupling operators. For pure flux (longitudinal) noise we take

$$
\mathcal{A}^{(\mathrm{flux})}=2 \pi \alpha \sin \left(2 \pi f+2 \varphi_{m}\right),
$$

which in the case of the subspace of the two lowest levels corresponds to $\mathcal{A} \approx-\lambda_{f} \sigma_{z}$, with $\lambda_{f}=2 \pi \alpha \mid\langle+| \sin (2 \pi f+$ $\left.2 \varphi_{m}\right)|+\rangle \mid \approx 4.5$, for FQ parameters $\alpha=0.8$ and $\eta=0.25$.

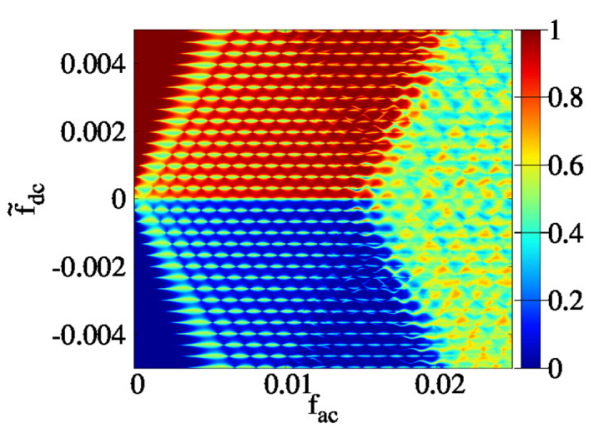

(a)

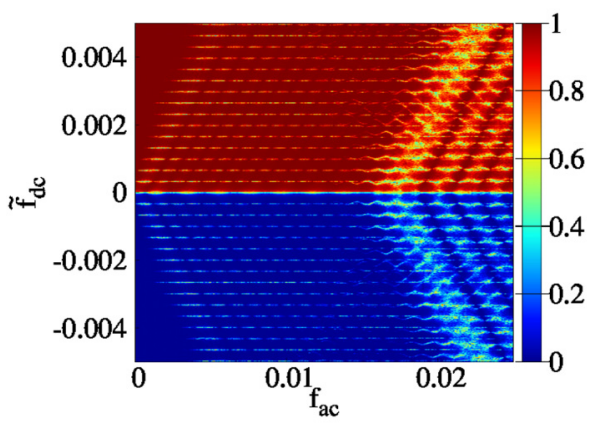

(b)

FIG. 5. LZS interference patterns. Plots of $P_{+}$as a function of the driving amplitude $f_{\text {ac }}$ and dc detuning $\tilde{f}_{\mathrm{dc}}$ for $t=1000 \tau$. (a) Flux noise. (b) Charge noise. The calculations were performed for $\omega_{0}=$ $2 \pi / \tau=0.003 E_{J} / \hbar$, Ohmic bath at $T=0.0014 E_{J} / k_{B} \sim 20 \mathrm{mK}$, and $\gamma=0.001$ (see text for details).

In the case of charge (transverse) noise the system operator is

$$
\mathcal{A}^{\text {(charge) }}=n_{m}=i \frac{\eta^{2}}{2(1+2 \alpha)} \frac{\partial}{\partial \varphi_{m}},
$$

which for the subspace of the two lowest levels gives $\mathcal{A} \approx$ $-\lambda_{c h} \sigma_{y}$, with $\lambda_{c h}=\frac{\eta^{2}}{4+8 \alpha}\left|\left\langle-\left|n_{m}\right|+\right\rangle\right| \approx 3 \times 10^{-4}$ for the same FQ parameters.

Notice that, after introducing realistic parameters, we obtain $\lambda_{c h} \ll \lambda_{f}$. For this parameter value, since $\left(\lambda^{(c h)} / \lambda^{(f)}\right)^{2} \sim$ $10^{-8} \ll 0.005$, it is irrelevant to study the mixed dynamics with both types of couplings since the transverse noise effects will be unobserved. Thus, we will consider only the cases of pure longitudinal and pure transverse noise in this section to analyze the effect of each type of noise on the LZS patterns separately. In Fig. 5 we plot $P_{+}$as a function of the driving amplitude $f_{\mathrm{ac}}$ and dc detuning $\tilde{f}_{\mathrm{dc}}$ for $t_{\mathrm{exp}}=1000 \tau$, and in Fig. 6 we plot it for the steady state. The LZS interference patterns show the typical "diamond" structure for increasing $f_{\text {ac }}$, concomitant with the additional transitions at the avoided crossings between different energy levels $[8,31,32]$. We plot a range of $f_{\text {ac }}$ that shows the first LZS diamond, D1, and the lower half of the second LZS diamond, D2. D1 can be described in terms of the dynamics of the two lowest energy levels; the region between D1 and D2 involves the dynamics of the three lowest energy levels, while D2 includes the four lowest energy levels (see Ref. [8] for a complete description of the multilevel LZS diamonds). 


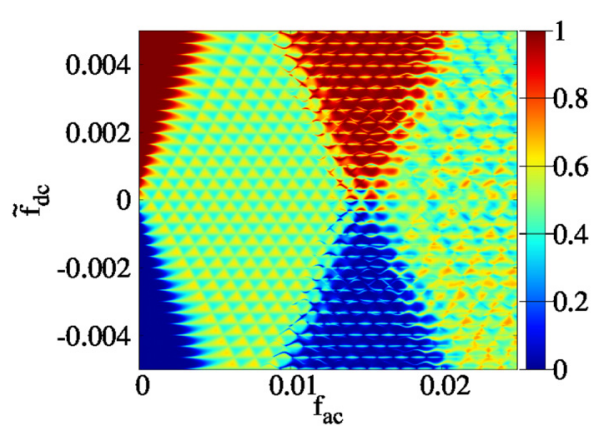

(a)

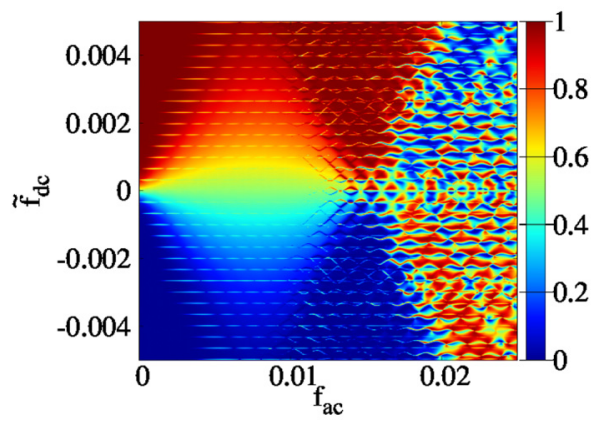

(b)

FIG. 6. LZS interference patterns. Plots of $P_{+}$as a function of the driving amplitude $f_{\mathrm{ac}}$ and dc detuning $\tilde{f}_{\mathrm{dc}}$ for the asymptotic regime, $t \rightarrow \infty$. (a) Flux noise. (b) Charge noise. Parameters are the same as in Fig. 5.

For finite time $t \sim t_{\exp }$, symmetric resonance lobes are observed within D1 for both types of coupling. However, for the transverse coupling case [Fig. 5(b)] the resonance lobes are narrower than for the longitudinal coupling [Fig. 5(a)]. The width of the resonance peaks is roughly proportional to the decoherence rate $1 / t_{d}[5,6]$. As analyzed in the previous section, in the transverse case dephasing mechanisms vanish out of resonance, and $1 / t_{d}$ is minimum. On the other hand, the dephasing rate grows out of resonance in the longitudinal case, and $1 / t_{d}$ is large.

In the steady state the differences among the two types of coupling are stronger. However, for the longitudinal coupling, Fig. 6(a) shows the triangular checkerboard pattern characteristic of antisymmetric resonances together with population inversion (both features are described in detail in Refs. [31,32]); for the transverse coupling [Fig. 6(b)], D1 exhibits a predominant background with a symmetric lobe in $P_{+}$around $\tilde{f_{\mathrm{dc}}}=0$. Within D2 and for the longitudinal coupling case, the patterns look qualitatively similar at finite time $t \sim t_{\exp }$ and in the steady state, respectively. On the other hand, for the transverse coupling case, the steady-state profile shows a strong population inversion in $\mathrm{D} 2$, absent at finite time $t \sim t_{\text {exp }}$.

To understand the different timescales, we plot $P_{+}$at a finite time and in the steady state as a function of the driving amplitude $f_{\text {ac }}$ for a fixed off-resonant value of detuning $\tilde{f}_{\mathrm{dc}}=$ $0.0009 \equiv 2.7 f_{\omega}$ for the longitudinal coupling [Fig. 7(a)] and for the transverse coupling [Fig. 7(b)]. The timescales of decoherence and relaxation, $t_{d}$ and $t_{r}$, are plotted in Fig. 7(c). In the previous section we concluded that for same coupling strengths, $\lambda_{c h}=\lambda_{f}$, the transverse coupling leads to a faster

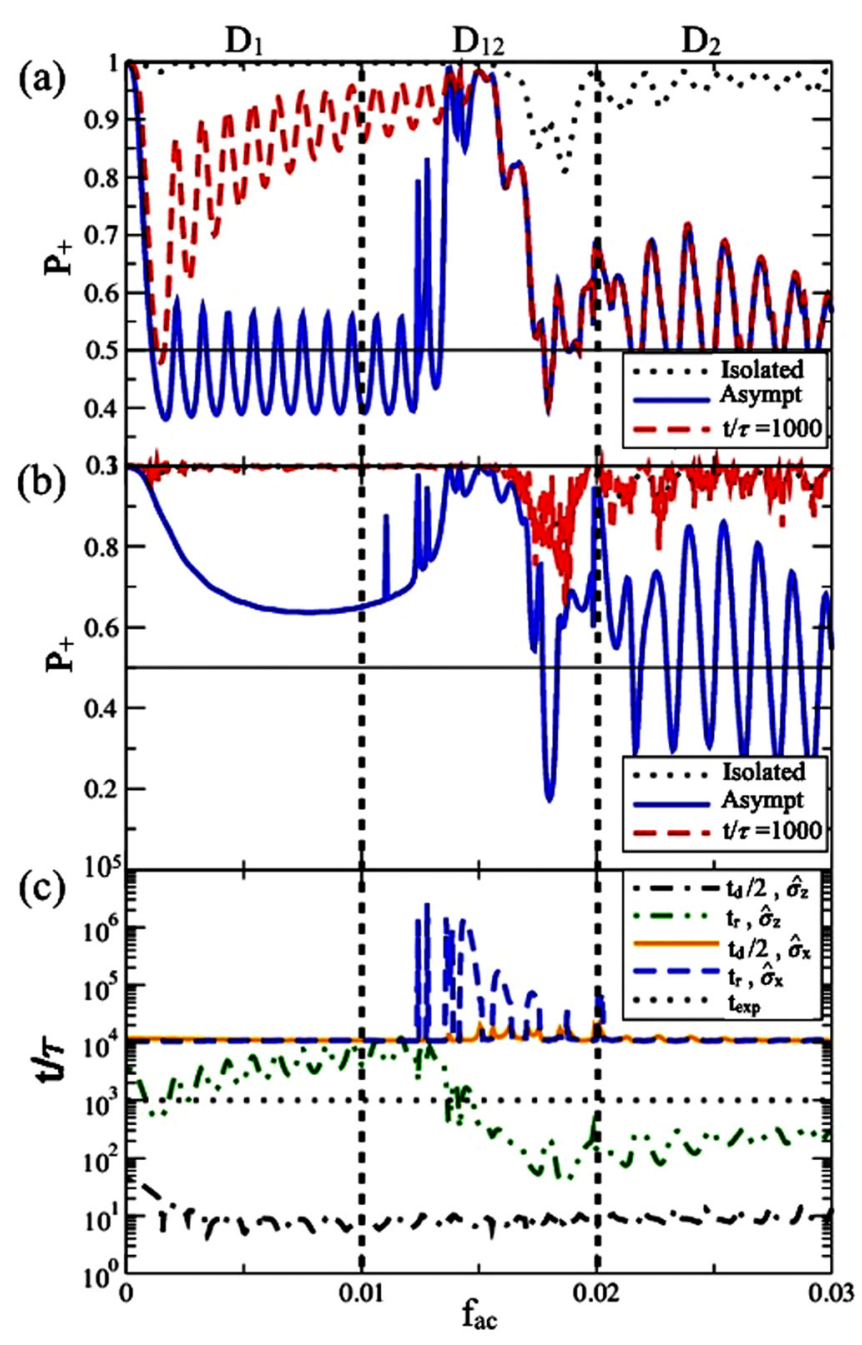

FIG. 7. $P_{+}$vs $f_{\mathrm{ac}}$ for $\tilde{f}_{\mathrm{dc}} \equiv 2.7 f_{\omega}$ for $t=1000 \tau$ (red dashed line), for the asymptotic state $t \rightarrow \infty$, (blue solid line), and for the isolated case (dots). (a) Flux noise. (b) Charge noise. (c) $t_{r} / \tau$ for flux noise (dash-double-dotted line) and charge noise (dashed line), $t_{d} / 2 \tau$ for flux noise (dot-dashed line) and charge noise (solid line), and $t_{\exp }=$ $1000 \tau$ (dotted line). The vertical dashed lines are guides for the eyes to show the boundaries of diamonds D1 and D2 and the region in between, named D12, for the value of $\tilde{f}_{\mathrm{dc}} \equiv 2.7 f_{\omega}$.

relaxation rate. Here the smallness of $\lambda_{c h}$ gives a much larger $t_{r}$ than in the $\lambda_{c h} \sim 1$ case analyzed previously. It is interesting to note in Fig. 7(c) that the resulting $t_{r}$ for the transverse coupling turns out to be of the same order or larger than in the longitudinal case.

From Fig. 7(c) it follows that for the longitudinal case and within D1, there is a large timescale separation $t_{d} \ll t_{\exp } \ll t_{r}$, in agreement with the different behaviors of $P_{+}\left(t_{\exp }=1000 \tau\right)$ and $P_{+}(\infty)$ seen in Fig. 7(a). The relaxation time strongly depends on $f_{\text {ac }}$, and within $\mathrm{D} 2, t_{r}$ is reduced two orders of magnitude, leading to $t_{r}<t_{\exp }$, and therefore, $P_{+}\left(t_{\exp }\right) \approx$ $P_{+}(\infty)$.

For the transverse coupling case [see Fig. 7(b)], within both diamonds D1 and D2 the timescales $t_{r}$ and $t_{d}$ are both larger than $t_{\exp }=1000 \tau$ and nearly independent of $f_{\text {ac }}$ (except in the transition between D1 and D2). Thus, for this type of coupling the steady-state behavior could not be seen at the 
experimental timescale either for D1 or for D2. Furthermore, it is also evident that the decoherence rate is minimum since $t_{r} \sim t_{d} / 2$ in the whole range of $f_{\text {ac }}$, even beyond the two-level regime discussed in the previous section.

From our analysis it is clear that the experimental results of Refs. [6,9] do not correspond to any of the steady-state LZS patterns of Fig. 6 since these experiments do not show either the antisymmetric resonance patterns of the longitudinal coupling or the background lobe for the off-resonant population of the transverse coupling. In addition, the extremely narrow resonance lobes of Fig. 5(b) for the transverse coupling do not seem to represent well the experimental data. The symmetric resonance lobes of the experimental LZS patterns are more in agreement with the case in Fig. 5(a) for longitudinal coupling. This conclusion is consistent with the noise spectroscopy measurements of Refs. [21-23] that found that the transverse noise is very small for FQ devices.

\section{CONCLUDING REMARKS}

We have performed a systematic analysis of environmental noise effects for a strongly driven FQ device, considering a realistic multilevel dynamics and emphasizing the behavior at different timescales.

A main outcome of our work is exposing the LZS interferometry as a tool to unveil the type of system-bath coupling, where the presence of symmetric (asymmetric) $n$-photon resonances in the stationary patterns reveals the nature of the noise, i.e., transverse (longitudinal) system-bath coupling.

In addition the analysis of the relaxation and decoherence timescales shows that the ratio $t_{r} / 2 t_{d}$ is also extremely sensitive to the type of system-bath coupling and might change significantly when an $n$-photon resonance is tuned.

For timescales prior to relaxation, the LZS interferometric patterns also exhibit two well-differentiated behaviors depending on the noise sources. Along this line, our results for the FQ device in the regime of strong driving (beyond the TLS regime) shed light on the interpretation of the experimental LZS diamonds obtained in Refs. [8,9] for a driven FQ with long relaxation times. The symmetric resonance lobes observed in Refs. [8,9] are in agreement with the longitudinal noise scenario shown in Fig. 5(a). However, to conclusively discard other possible scenarios, experiments should be performed for larger driving times in order to reach the steady state after full relaxation with the bath degrees of freedom.

Experimental studies of noise spectroscopy for the FQ, when driven at the first resonance, have shown that flux noise is the dominant source of decoherence [22,23]. This result is also consistent with the scenario of longitudinal noise found in Fig. 5(a) for the case of multiphoton resonances and large amplitudes. However, the flux noise power spectrum at low frequencies has shown $1 / f$ behavior [21-23]. Thus, to better account for noise effects in the steady state or long-time limit, future studies based on a non-Markovian description [64] would be interesting.

Even when we have considered specific parameters of the FQ, our results can also be useful for other qubits and artificial atom devices, in which the amplitude spectroscopy technique based on LZS interferometry was implemented in recent years [11,14-16,48,54].

\section{ACKNOWLEDGMENTS}

We acknowledge financial support from CNEA, CONICET (Grant No. PIP11220150100756), UNCuyo (Grant No. P 06/C455), and ANPCyT (Grants No. PICT2014-1382 and No. PICT2016-0791).

\section{APPENDIX A: THE ROTATING WAVE APPROXIMATION: DRESSED BASIS}

In this Appendix we briefly revisit the rotating-wave approximation (RWA) applied to multiphoton resonances $[5,12,42,65]$. We start by considering the general two-level system (TLS) Hamiltonian:

$$
\mathcal{H}_{\mathrm{TLS}}(t)=-\frac{\epsilon(t)}{2} \sigma_{z}-\frac{\Delta}{2} \sigma_{x},
$$

where $\epsilon(t)=\epsilon_{0}+A \cos \left(\omega_{0} t\right)$. The parameter $\epsilon_{0}$ is the polarization energy of the qubit; $A$ and $\omega$ are the amplitude and frequency of the driving, respectively. By applying the unitary transformation $|\tilde{\psi}(t)\rangle=U_{\phi}(t)|\psi(t)\rangle$, with $U_{\phi}(t)=e^{-i \frac{\phi(t)}{2} \sigma_{z}}$ and $\phi(t)=\int \epsilon(t) d t=\epsilon_{0} t+A / \omega_{0} \sin \left(\omega_{0} t\right)$, the transformed Hamiltonian reads

$$
\tilde{H}=-\frac{[\epsilon-\dot{\phi}(t)]}{2} \sigma_{z}-\frac{\Delta}{2}\left(e^{-i \phi(t)} \sigma_{+}+\text {H.c. }\right) .
$$

Replacing $\quad \phi(t) \rightarrow \phi_{n}(t)=n \omega_{0} t+\frac{A}{\omega_{0}} \sin \omega_{0} t \quad$ (which is equivalent to taking the resonance condition $\left.\epsilon_{0} \sim n \omega_{0}\right)$, the Hamiltonian transforms to

$$
\tilde{H}=-\frac{\left(\epsilon_{0}-n \omega_{0}\right)}{2} \sigma_{z}-\frac{\Delta}{2}\left(e^{-i n \omega_{0} t} e^{-i \frac{A}{\omega_{0}} \sin \omega_{0} t} \sigma_{+}+\text {H.c. }\right) \text {. }
$$

Using in addition that $e^{i x \sin a}=\sum_{k=-\infty}^{k=+\infty} J_{k}(x) e^{i k a}$, with $J_{k}(x)$ being the Bessel function of order $k$, we can write

$$
e^{i n \omega_{0} t} e^{i \frac{A}{\omega_{0}} \sin \omega_{0} t}=\sum_{k=-\infty}^{k=+\infty} J_{k}\left(\frac{A}{\omega_{0}}\right) e^{i(k+n) \omega_{0} t} \simeq J_{-n}\left(\frac{A}{\omega_{0}}\right),
$$

where in the last step we have performed a RWA, for $\mid \epsilon_{0}-$ $n \omega_{0} \mid \ll \Delta$. In this way, we finally obtain the TLS Hamiltonian written in the RWA as

$$
\tilde{H} \simeq \tilde{H}_{n}=-\frac{\left(\epsilon_{0}-n \omega_{0}\right)}{2} \sigma_{z}-\frac{\Delta_{-n}}{2} \sigma_{x},
$$

with $\Delta_{-n}=\Delta J_{-n}\left(\frac{A}{\omega_{0}}\right)$.

Notice that after the RWA we have obtained an effective time-independent "dressed" Hamiltonian. Going a step further, we proceed to diagonalize $\tilde{H}_{n}$ considering the operator $U_{r}=\cos (\varphi) \sigma_{z}+\sin (\varphi) \sigma_{x}$. After applying such a transformation, we finally obtain the dressed Hamiltonian

$$
H_{r}=U_{r} \tilde{H}_{n} U_{r}^{-1}=-\frac{\Omega_{n}}{2} \sigma_{z},
$$

with $\cos (2 \varphi)=\epsilon_{n} / \Omega_{n}, \sin (2 \varphi)=\Delta_{-n} / \Omega_{n}, \epsilon_{n}=\epsilon_{0}-n \omega_{0}$, and $\Omega_{n}=\sqrt{\left(\epsilon_{0}-n \omega_{0}\right)^{2}+\Delta_{-n}^{2}}$ being the generalized Rabi frequency. It is worth noting that the eigenenergies of $H_{r}$ exhibit an avoided crossing with an effective dressed gap $\Delta_{-n}$, and the associated eigenstates form the dressed basis. 


\section{APPENDIX B: CALCULATION OF RELAXATION AND DECOHERENCE RATES IN THE ROTATING WAVE APPROXIMATION}

The dynamics of an open system can be described by the total Hamiltonian:

$$
H_{T}(t)=\mathcal{H}_{\mathrm{TLS}}(t)+H_{B}+H_{\mathrm{int}},
$$

where $\mathcal{H}_{\mathrm{TLS}}(t)$ is the driven TLS Hamiltonian, $H_{B}$ is the bath term, and

$$
H_{\text {int }}=\mathcal{A} \otimes \mathcal{B}
$$

is the system-bath interaction term. In the present analysis the system operator $\mathcal{A}$ can be $\mathcal{A}_{z}=\lambda_{z} \sigma_{z}$ or $\mathcal{A}_{x}=\lambda_{x} \sigma_{x}$, and $\mathcal{B}$ is the bath operator.

The von Neumann equation for time evolution of the system described by the total Hamiltonian (B1) is (taking $\hbar=1)$

$$
\frac{\partial \rho_{T}(t)}{\partial t}=-i\left[H_{T}(t), \rho_{T}(t)\right],
$$

with $\rho_{T}(t)$ being the density matrix of the global system.

We start by defining $H_{0}(t)=\mathcal{H}_{\mathrm{TLS}}(t)+H_{B}$, and the associated evolution operator $U_{0}(t)=\hat{\mathcal{T}} e^{-i \int H_{0}(t) d t}$. Therefore, in the interaction picture the transformed operators are $\tilde{\rho}(t)=$ $U_{0}^{\dagger}(t) \rho(t) U_{0}(t)$ and $\tilde{H}_{\text {int }}(t)=U_{0}^{\dagger}(t) H_{\text {int }} U_{0}(t)$, and Eq. (B3) reads

$$
\frac{\partial \tilde{\rho_{T}}(t)}{\partial t}=-i\left[\tilde{H}_{\mathrm{int}}(t), \tilde{\rho_{T}}(t)\right] .
$$

After defining the system reduced density matrix $\rho=$ $\operatorname{Tr}_{B}\left(\rho_{T}\right)$ and performing the Born-Markov approximation, we get

$$
\begin{aligned}
\frac{\partial \tilde{\rho}}{\partial t}= & -\int_{0}^{\infty} d t^{\prime}\left\{G\left(t^{\prime}\right)\left[\tilde{\mathcal{A}}(t) \tilde{\mathcal{A}}\left(t-t^{\prime}\right) \tilde{\rho}-\tilde{\mathcal{A}}\left(t-t^{\prime}\right) \tilde{\rho} \tilde{\mathcal{A}}(t)\right]\right. \\
& \left.+G\left(-t^{\prime}\right)\left[\tilde{\rho} \tilde{\mathcal{A}}\left(t-t^{\prime}\right) \tilde{\mathcal{A}}(t)-\tilde{\mathcal{A}}(t) \tilde{\rho} \tilde{\mathcal{A}}\left(t-t^{\prime}\right)\right]\right\}, \quad(\mathrm{B} 5
\end{aligned}
$$

with $G(t)=\operatorname{Tr}_{B}\left[\mathcal{B}(t) \mathcal{B}(0) \rho_{B}\right]$ being the bath correlation function and $\tilde{\mathcal{A}}(t)=U_{0}^{\dagger}(t) \mathcal{A} U_{0}(t)$.

Now, we set the decomposition

$$
\tilde{\mathcal{A}}(t)=\sum_{\omega} e^{-i \omega t} P_{\omega}=\sum_{\omega} e^{i \omega t} P_{\omega}^{\dagger}=\sum_{\omega>0} e^{-i \omega t} P_{\omega}+e^{i \omega t} P_{\omega}^{\dagger},
$$

with $P_{-\omega}=P_{\omega}^{\dagger}$. Moreover, we can define $\Gamma(\omega)=$ $\int_{0}^{\infty} d t e^{-i \omega t} G(t)=\frac{1}{2} g(\omega)+i s(\omega)[66]$.

After performing the secular approximation, Eq. (B5) can be expressed in the Lindblad form as

$$
\frac{\partial \tilde{\rho}}{\partial t}=-i\left[H_{L}, \tilde{\rho}\right]+\sum_{\omega} g(\omega)\left(P_{\omega} \tilde{\rho} P_{\omega}^{\dagger}-\frac{1}{2}\left\{P_{\omega}^{\dagger} P_{\omega}, \tilde{\rho}\right\}\right),
$$

with the Hamiltonian

$$
H_{L}=\sum_{\omega} s(\omega) P_{\omega}^{\dagger} P_{\omega} .
$$

We now proceed to transform the system operator $\mathcal{A}$ into the dressed representation [12], $\mathcal{A} \rightarrow \mathcal{A}_{r}$. Following the procedure described previously, we perform the transformation $\mathcal{A}_{r}(t)=U_{r} U_{n} \mathcal{A} U_{n}^{-1} U_{r}^{-1}$, with $U_{r}=\cos (\varphi) \sigma_{z}+\sin (\varphi) \sigma_{x}$ and $U_{n}(t)=e^{-i \frac{1}{2} \phi_{n}(t) \sigma_{z}}$.
For a system operator of the form $\mathcal{A}=\lambda\left(\cos \theta \sigma_{z}+\right.$ $\sin \theta \sigma_{x}$ ), we obtain the transformed $\mathcal{A}_{r}$ as

$$
\mathcal{A}_{r}(t)=a_{x}(t) \sigma_{x}+a_{y}(t) \sigma_{y}+a_{z}(t) \sigma_{z},
$$

with the coefficients $a_{i}(t), i=x, y, z$, satisfying the following relations:

$$
\begin{aligned}
& a_{x}(t)=a_{x 0}+a_{x c} \cos \phi_{n}(t) \approx a_{x 0}+a_{x c} c_{0}, \\
& a_{y}(t)=a_{y s} \sin \phi_{n}(t) \approx 0, \\
& a_{z}(t)=a_{z 0}+a_{z c} \cos \phi_{n}(t) \approx a_{z 0}+a_{z c} c_{0},
\end{aligned}
$$

with $\quad a_{x 0}=\lambda \cos \theta \sin 2 \varphi, a_{x c}=-\lambda \sin \theta \cos 2 \varphi, a_{y s}=$ $-\lambda \sin \theta, a_{z 0}=\lambda \cos \theta \cos 2 \varphi$, and $a_{z c}=\lambda \sin \theta \sin 2 \varphi$. In the last step we have performed the RWA as in Eq. (A4), with $c_{0}=J_{-n}\left(\frac{A}{\omega_{0}}\right)$.

Transforming $\mathcal{A}_{r}$ into the interaction picture, one gets

$$
\begin{aligned}
\tilde{\mathcal{A}}_{r}(t)= & \left(a_{x 0}+a_{x c} c_{0}\right) e^{i \Omega_{n} t} \sigma_{+}+\left(a_{x 0}+a_{x c} c_{0}\right) e^{-i \Omega_{n} t} \sigma_{-} \\
& +\left(a_{z 0}+a_{z c} c_{0}\right) \sigma_{z} .
\end{aligned}
$$

To obtain the Lindblad equation, we rewrite the above equation in terms of the decomposition of Eq. (B6),

$$
\begin{aligned}
\tilde{\mathcal{A}}_{r}(t) & =P_{+}\left(\Omega_{n}\right) e^{i \Omega_{n} t}+P_{-}\left(-\Omega_{n}\right) e^{-i \Omega_{n} t}+P_{0} \\
& =P_{+}\left(\Omega_{n}\right) e^{i \Omega_{n} t}+P_{+}^{\dagger}\left(\Omega_{n}\right) e^{-i \Omega_{n} t}+P_{0} \\
& =\sum_{\omega} P_{\omega} e^{i \omega t},
\end{aligned}
$$

with $\omega \in\left\{0, \Omega_{n},-\Omega_{n}\right\} \quad$ and $\quad P_{\omega}=\left\{P_{z}(0), P_{+}\left(\Omega_{n}\right)\right.$, $\left.P_{-}\left(-\Omega_{n}\right)\right\} \equiv\left\{P_{z}(0), P_{+}\left(\Omega_{n}\right), P_{+}^{\dagger}\left(\Omega_{n}\right)\right\}$. The operators $P_{\omega}$ are

$$
\begin{gathered}
P_{z}(0)=\left(a_{z 0}+a_{z c} c_{0}\right) \sigma_{z}=z(0) \sigma_{0}, \\
P_{+}\left(\Omega_{n}\right)=\left(a_{x 0}+a_{x c} c_{0}\right) \sigma_{+}=x\left(\Omega_{n}\right) \sigma_{+}, \\
P_{-}\left(-\Omega_{n}\right)=P_{+}^{\dagger}\left(\Omega_{n}\right)=x\left(-\Omega_{n}\right) \sigma_{-},
\end{gathered}
$$

with $z(0)=a_{z 0}+a_{z c} c_{0}$ and $x\left(\Omega_{n}\right)=a_{x 0}+a_{x c} c_{0}$.

Using Eq. (B12) in Eq. (B10), we obtain the Lindblad equation

$$
\begin{aligned}
\frac{\partial \tilde{\rho}}{\partial t}= & -\Gamma_{\uparrow}\left(\begin{array}{cc}
-\tilde{\rho}_{11} & \frac{1}{2} \tilde{\rho}_{12} \\
\frac{1}{2} \tilde{\rho}_{21} & \tilde{\rho}_{11}
\end{array}\right)-\Gamma_{\downarrow}\left(\begin{array}{cc}
\tilde{\rho}_{22} & \frac{1}{2} \tilde{\rho}_{12} \\
\frac{1}{2} \tilde{\rho}_{21} & -\tilde{\rho}_{22}
\end{array}\right) \\
& -\Gamma_{0}\left(\begin{array}{cc}
0 & \tilde{\rho}_{12} \\
\tilde{\rho}_{21} & 0
\end{array}\right),
\end{aligned}
$$

with

$$
\begin{aligned}
& \Gamma_{\uparrow}=\left|x\left(\Omega_{n}\right)\right|^{2} g\left(-\Omega_{n}\right), \\
& \Gamma_{\downarrow}=\left|x\left(\Omega_{n}\right)\right|^{2} g\left(\Omega_{n}\right), \\
& \Gamma_{o}=|z(0)|^{2} g(0) .
\end{aligned}
$$

After solving Eq. (B13), the relaxation $\Gamma_{r}$ and decoherence $\Gamma_{d}$ rates can be computed as

$$
\begin{aligned}
& \Gamma_{d}=\Gamma_{\downarrow}+\Gamma_{\uparrow}, \\
& \Gamma_{r}=\Gamma_{0}+\frac{\Gamma_{d}}{2} .
\end{aligned}
$$


Considering the system-bath coupling term $\mathcal{A}_{z}=\lambda_{z} \sigma_{z}$, the rates in Eq. (B15) take the form

$$
\begin{aligned}
& \Gamma_{\uparrow}^{z}=\left|\lambda_{z} \sin (2 \varphi)\right|^{2} g\left(-\Omega_{n}\right), \\
& \Gamma_{\downarrow}^{z}=\left|\lambda_{z} \sin (2 \varphi)\right|^{2} g\left(\Omega_{n}\right), \\
& \Gamma_{o}^{z}=\left|\lambda_{z} \cos (2 \varphi)\right|^{2} g(0),
\end{aligned}
$$

followed by

$$
\begin{aligned}
& \Gamma_{d}^{z}=\left|\lambda_{z} \sin (2 \varphi)\right|^{2}\left[g\left(-\Omega_{n}\right)+g\left(\Omega_{n}\right)\right], \\
& \Gamma_{r}^{z}=\left|\lambda_{z} \cos (2 \varphi)\right|^{2} g(0)+\frac{\Gamma_{d}^{z}}{2} .
\end{aligned}
$$

For the $\mathcal{A}_{x}=\lambda_{x} \sigma_{x}$ case, the rates are

$$
\begin{aligned}
\Gamma_{\uparrow}^{x} & =\left|\lambda_{x} c_{0} \cos (2 \varphi)\right|^{2} g\left(-\Omega_{n}\right), \\
\Gamma_{\downarrow}^{x} & =\left|\lambda_{x} c_{0} \cos (2 \varphi)\right|^{2} g\left(\Omega_{n}\right), \\
\Gamma_{o}^{x} & =\left|\lambda_{x} c_{0} \sin (2 \varphi)\right|^{2} g(0) .
\end{aligned}
$$

For this case, the calculation of the rates $\Gamma_{d}^{x}$ and $\Gamma_{r}^{x}$ is rather cumbersome. We obtain

$$
\begin{aligned}
& \Gamma_{d}^{x}=\left|\lambda_{x} c_{0} \cos (2 \varphi)\right|^{2}\left[g\left(-\Omega_{n}\right)+g\left(\Omega_{n}\right)\right], \\
& \Gamma_{r}^{x}=\left|\lambda_{x} c_{0} \sin (2 \varphi)\right|^{2} g(0) .
\end{aligned}
$$

In this way, we have extended the calculation of relaxation rates given in the Supplementary Information of [22] to the case of $n$-photon resonances.
[1] T. P. Orlando, J. E. Mooij, L. Tian, C. H. van der Wal, L. S. Levitov, S. Lloyd, and J. J. Mazo, Phys. Rev. B 60, 15398 (1999).

[2] I. Chiorescu, Y. Nakamura, C. J. P. M. Harmans, and J. E. Mooij, Science 299, 1869 (2003).

[3] J. Q. You and F. Nori, Nature (London) 474, 589 (2011).

[4] Y. Makhlin, G. Schön, and A. Shnirman, Rev. Mod. Phys. 73, 357 (2001).

[5] S. Shevchenko, S. Ashhab, and F. Nori, Phys. Rep. 492, 1 (2010).

[6] W. D. Oliver, Y. Yu, J. C. Lee, K. K. Berggren, L. S. Levitov, and T. P. Orlando, Science 310, 1653 (2005).

[7] D. M. Berns, W. D. Oliver, S. O. Valenzuela, A. V. Shytov, K. K. Berggren, L. S. Levitov, and T. P. Orlando, Phys. Rev. Lett. 97, 150502 (2006).

[8] D. M. Berns, M. S. Rudner, S. O. Valenzuela, K. K. Berggren, W. D. Oliver, L. S. Levitov, and T. P. Orlando, Nature (London) 455, 51 (2008).

[9] W. D. Oliver and S. O. Valenzuela, Quantum Inf. Process. 8, 261 (2009).

[10] A. Izmalkov, S. H. W. van der Ploeg, S. N. Shevchenko, M. Grajcar, E. Il'ichev, U. Hübner, A. N. Omelyanchouk, and H.G. Meyer, Phys. Rev. Lett. 101, 017003 (2008).

[11] M. Sillanpää, T. Lehtinen, A. Paila, Y. Makhlin, and P. Hakonen, Phys. Rev. Lett. 96, 187002 (2006).

[12] C. M. Wilson, T. Duty, F. Persson, M. Sandberg, G. Johansson, and P. Delsing, Phys. Rev. Lett. 98, 257003 (2007).

[13] S. Yoakum, L. Sirko, and P. M. Koch, Phys. Rev. Lett. 69, 1919 (1992).

[14] M. Mark, T. Kraemer, P. Waldburger, J. Herbig, C. Chin, H.-C. Nägerl, and R. Grimm, Phys. Rev. Lett. 99, 113201 (2007).

[15] S. Kling, T. Salger, C. Grossert, and M. Weitz, Phys. Rev. Lett. 105, 215301 (2010).

[16] P. Huang, J. Zhou, F. Fang, X. Kong, X. Xu, C. Ju, and J. Du, Phys. Rev. X 1, 011003 (2011).

[17] F. Forster, G. Petersen, S. Manus, P. Hänggi, D. Schuh, W. Wegscheider, S. Kohler, and S. Ludwig, Phys. Rev. Lett. 112, 116803 (2014).

[18] R. Blattmann, P. Hänggi, and S. Kohler, Phys. Rev. A 91, 042109 (2015).
[19] X. Mi, S. Kohler, and J. R. Petta, Phys. Rev. B 98, 161404(R) (2018).

[20] F. Yan, S. Gustavsson, A. Kamal, J. Birenbaum, A. P. Sears, D. Hover, T. J. Gudmundsen, D. Rosenberg, G. Samach, S. Weber, J. L. Yoder, T. P. Orlando, J. Clarke, A. J. Kerman, and W. D. Oliver, Nat. Commun. 7, 12964 (2016).

[21] J. Bylander, S. Gustavsson, F. Yan, F. Yoshihara, K. Harrabi, G. Fitch, D. G. Cory, Y. Nakamura, J.-S. Tsai, and W. D. Oliver, Nat. Phys. 7, 565 (2011).

[22] F. Yan, S. Gustavsson, J. Bylander, X. Jin, F. Yoshihara, D. G. Cory, Y. Nakamura, T. P. Orlando, and W. D. Oliver, Nat. Commun. 4, 2337 (2013).

[23] F. Yoshihara, Y. Nakamura, F. Yan, S. Gustavsson, J. Bylander, W. D. Oliver, and J.-S. Tsai, Phys. Rev. B 89, 020503(R) (2014).

[24] M. Grifoni and P. Hänggi, Phys. Rep. 304, 229 (1998).

[25] S. Kohler, R. Utermann, P. Hänggi, and T. Dittrich, Phys. Rev. E 58, 7219 (1998).

[26] L. Hartmann, I. Goychuk, M. Grifoni, and P. Hänggi, Phys. Rev. E 61, R4687(R) (2000).

[27] Y. Dakhnovskii, D. G. Evans, H. J. Kim, and R. D. Coalson, J. Chem. Phys. 103, 5461 (1995).

[28] T. M. Stace, A. C. Doherty, and S. D. Barrett, Phys. Rev. Lett. 95, 106801 (2005).

[29] M. C. Goorden, M. Thorwart, and M. Grifoni, Phys. Rev. Lett. 93, 267005 (2004).

[30] J. Hausinger and M. Grifoni, Phys. Rev. A 81, 022117 (2010).

[31] A. Ferrón, D. Domínguez, and M. J. Sánchez, Phys. Rev. Lett. 109, 237005 (2012).

[32] A. Ferrón, D. Domínguez, and M. J. Sánchez, Phys. Rev. B 93, 064521 (2016).

[33] A. Ferrón and D. Domínguez, Phys. Rev. B 81, 104505 (2010).

[34] J. Q. You, X. Hu, S. Ashhab, and F. Nori, Phys. Rev. B 75, 140515(R) (2007).

[35] E. A. Sete, M. J. Reagor, N. Didier, and C. T. Rigetti, Phys. Rev. Appl. 8, 024004 (2017).

[36] For the qubit parameters under consideration, one can show that the ratio of matrix elements of the charge operators for the lowest-energy states is $\frac{\left|\left\langle i\left|\hat{n}_{p}\right| j\right\rangle\right|}{\left\langle\left\langle i\left|\hat{n}_{m}\right| j\right\rangle\right|} \sim 10^{-10}$.

[37] M. S. Rudner, A. V. Shytov, L. S. Levitov, D. M. Berns, W. D. Oliver, S. O. Valenzuela, and T. P. Orlando, Phys. Rev. Lett. 101, 190502 (2008). 
[38] J. H. Shirley, Phys. Rev. 138, B979 (1965).

[39] H.-P. Breuer, W. Huber, and F. Petruccione, Phys. Rev. E 61, 4883 (2000).

[40] D. W. Hone, R. Ketzmerick, and W. Kohn, Phys. Rev. E 79, 051129 (2009).

[41] A. Ferrón, D. Domínguez, and M. J. Sánchez, Phys. Rev. B 82, 134522 (2010).

[42] S. Ashhab, J. R. Johansson, A. M. Zagoskin, and F. Nori, Phys. Rev. A 75, 063414 (2007).

[43] M. C. Goorden, M. Thorwart, and M. Grifoni, Eur. Phys. J. B 45, 405 (2005).

[44] C. H. van der Wal, F. K. Wilhelm, C. J. P. M. Harmans, and J. E. Mooij, Eur. Phys. J. B 31, 111 (2003).

[45] Notice that when the different noise sources are fully correlated, such that $\mathcal{B}^{v}(t)=a_{v} \mathcal{B}(t)$, it is straightforward to show (after redefining $\mathcal{A}=\sum_{v} a_{v} \mathcal{A}^{v}$ ) that $H_{\text {int }}=\mathcal{A} \otimes \mathcal{B}$; that is, there is an effective single-noise source.

[46] F. Grossmann, T. Dittrich, P. Jung, and P. Hänggi, Phys. Rev. Lett. 67, 516 (1991).

[47] Y. Kayanuma and K. Saito, Phys. Rev. A 77, 010101(R) (2008).

[48] C. M. Wilson, G. Johansson, T. Duty, F. Persson, M. Sandberg, and P. Delsing, Phys. Rev. B 81, 024520 (2010).

[49] G. Sun, X. Wen, Y. Wang, S. Cong, J. Chen, L. Kang, W. Xu, Y. Yu, S. Han, and P. Wu, Appl. Phys. Lett. 94, 102502 (2009).

[50] G. Sun, X. Wen, B. Mao, Y. Yu, J. Chen, W. Xu, L. Kang, P. Wu, and S. Han, Phys. Rev. B 83, 180507(R) (2011).

[51] Y. Wang, S. Cong, X. Wen, C. Pan, G. Sun, J. Chen, L. Kang, W. Xu, Y. Yu, and P. Wu, Phys. Rev. B 81, 144505 (2010).
[52] S. E. de Graaf, J. Leppäkangas, A. Adamyan, A. V. Danilov, T. Lindström, M. Fogelström, T. Bauch, G. Johansson, and S. E. Kubatkin, Phys. Rev. Lett. 111, 137002 (2013).

[53] S. N. Shevchenko, A. N. Omelyanchouk, and E. Il'ichev, Low Temp. Phys. 38, 283 (2012).

[54] J. R. Petta, H. Lu, and A. C. Gossard, Science 327, 669 (2010).

[55] J. Stehlik, Y. Dovzhenko, J. R. Petta, J. R. Johansson, F. Nori, H. Lu, and A. C. Gossard, Phys. Rev. B 86, 121303(R) (2012).

[56] G. Cao, H.-O. Li, T. Tu, L. Wang, C. Zhou, M. Xiao, G.-C. Guo, H.-W. Jiang, and G.-P. Guo, Nat. Commun. 4, 1401 (2013).

[57] E. Dupont-Ferrier, B. Roche, B. Voisin, X. Jehl, R. Wacquez, M. Vinet, M. Sanquer, and S. De Franceschi, Phys. Rev. Lett. 110, 136802 (2013).

[58] R. Shang, H.-O. Li, G. Cao, M. Xiao, T. Tu, H. Jiang, G.-C. Guo, and G.-P. Guo, Appl. Phys. Lett. 103, 162109 (2013).

[59] P. Nalbach, J. Knörzer, and S. Ludwig, Phys. Rev. B 87, 165425 (2013).

[60] G. Granger, G. C. Aers, S. A. Studenikin, A. Kam, P. Zawadzki, Z. R. Wasilewski, and A. S. Sachrajda, Phys. Rev. B 91, 115309 (2015).

[61] J. Zhou, P. Huang, Q. Zhang, Z. Wang, T. Tan, X. Xu, F. Shi, X. Rong, S. Ashhab, and J. Du, Phys. Rev. Lett. 112, 010503 (2014).

[62] M. D. LaHaye, J. Suh, P. M. Echternach, K. C. Schwab, and M. L. Roukes, Nature (London) 459, 960 (2009).

[63] L. Du, M. Wang, and Y. Yu, Phys. Rev. B 82, 045128 (2010).

[64] T. M. Stace, A. C. Doherty, and D. J. Reilly, Phys. Rev. Lett. 111, 180602 (2013).

[65] M. A. Kmetic, R. A. Thuraisingham, and W. J. Meath, Phys. Rev. A 33, 1688 (1986).

[66] H.-P. Breuer and F. Petruccione, The Theory of Open Quantum Systems (Oxford University Press, New York, 2006). 